\title{
Reduction of Attributes in Averaged Similarities
}

\author{
D. Boixader J. Recasens
}

\begin{abstract}
Similarity Relations may be constructed from a set of fuzzy attributes. Each fuzzy attribute generates a simple similarity, and these simple similarities are combined into a complex similarity afterwards. The Representation Theorem establishes one such way of combining similarities, while averaging them is a different and more realistic approach in applied domains. In this paper, given an averaged similarity by a family of attributes, we propose a method to find families of new attributes having fewer elements that generate the same similarity. More generally, the paper studies the structure of this important class of fuzzy relations.
\end{abstract}

\section{Introduction}

Similarity Relations were introduced by Zadeh [11] as a natural way of fuzzifying classical equivalence relations. They have become widely used, and they appear under different names in the literature, depending on the authors and on the specific algebrization of the multivalued transitivity axiom. So Similarity Relation, Likeness, Fuzzy Equality, T-indistinguishability Operator, or simply Fuzzy Equivalence Relation, are common terms that refer to this class of fuzzy relations.

Following Trillas and Valverde [10] we favor the term T-indistinguishability Operator, which makes explicit reference to the $t$-norm $T$, and we will use Similarity only in an informal way.

Definition 1.1. Let $X$ be a set and $T$ a t-norm. A T-indistinguishability operator $E$ on $X$ is a fuzzy relation $E: X \times X \rightarrow[0,1]$ satisfying, for all $x, y, z \in X$, 
1. $E(x, x)=1$ (Reflexivity)

2. $E(x, y)=E(y, x)$ (Symmetry)

3. $T(E(x, y), E(y, z)) \leq E(x, z)$ (T-Transitivity)

The transitivity axiom involves a $t$-norm [6]. A $t$-norm $T$ is an operation on the unit interval that is associative, commutative, non-decreasing in both variables and which satisfies the boundary condition $T(x, 1)=x$ for all $x \in$ $[0,1]$. Since it is generally accepted that $t$-norms are the $A N D$ connectives of Fuzzy Logic [4], axiom 3 in Definition 1.1 can be read as if $x$ is similar to $y$, AND $y$ is similar to $z$, THEN $x$ is similar to $z$.

Definition 1.1 is intended to provide a formal framework for dealing with the primitive notion of similarity as perceived by humans. If, for example, an observer is presented with a set $X$ of characters written by hand by several people and questioned about the resemblances between them, such observer is likely to answer by providing a degree of similarity for each pair of handwritten characters. By normalizing those degrees a map $E: X \times X \rightarrow$ $[0,1]$ is obtained, which is expected to fulfill, whatever imperfectly, the axioms of a $T$-indistinguishability operator for some suitably chosen $t$-norm $T$.

On the other hand, the problem of determining the map (or fuzzy relation) $E$ may be tackled from an entirely different angle. We may select a set of criteria, such as size, thickness, roundness and so forth that every single character $x \in X$ is liable to fulfill to some degree. Again, by normalizing those degrees a family of fuzzy subsets $h_{i}: X \rightarrow[0,1], i \in I$, is obtained, each fuzzy subset representing one of the fuzzy criteria which have been selected as the features relevant to this problem. Such a family of fuzzy subsets induces a family of similarities on $X$, and some suitable combination of these similarities ought to yield, at least approximately, the map $E$ as a result.

In a theoretical setting, the Representation Theorem (2.5) [10] provides a synthesis of the two previous approaches which consists in taking the infima of all the elementary $T$-indistinguishability operators $E_{h_{i}}$ induced by single fuzzy subsets $h_{i}: X \rightarrow[0,1]$,

$$
E(x, y)=\inf _{i \in I} E_{h_{i}}(x, y)
$$

From an applied point of view, though, this way of aggregating the simple similarities induced by the attributes $h_{i}$ may seem a bit unrealistic. This is 
because a single attribute $h_{i}$ taking discordant values $h_{i}(x) \neq h_{i}(y)$ on two elements $x$ and $y$ is enough to render those elements strongly dissimilar by $E$, regardless of how many of the remaining attributes $h_{j}, j \neq i$, take close or even identical values on the pair. If the theory of similarity relations is to be applied to practical domains, some other ways of averaging the simple similarities $E_{h_{i}}$ need to be explored.

Quasi-arithmetic means (see [1]) are a family of averaging operators which are widely used. They may be easily related to the continuous Archimedean $t$-norms presented in Section 2, because they may also be obtained from additive generators. This common theoretical background makes them very suitable to average indistinguishabilities.

Across this paper we will be concerned with $T$-indistinguishability operators $\bar{E}$ such that

$$
\bar{E}=\underset{i \in I}{M}\left(\alpha_{i} E_{h_{i}}\right)
$$

where $M$ represents a quasi-arithmetic mean, $\left(\alpha_{i}\right)_{i \in I}$ a family of weights, and $T$ an Archimedean $t$-norm, although some particular results are also provided for the $t$-norm $T=M I N$. Our goal is to find alternative families $h_{j}^{\prime}: X \rightarrow[0,1], j \in J$, having fewer (and, in general, different) fuzzy attributes and such that

$$
\bar{E}=\underset{j \in J}{M}\left(\beta_{j} E_{h_{j}^{\prime}}\right) .
$$

Smaller generating families of fuzzy sets are of practical interest both for storage and for computational reasons, but also because they provide a way of representing data in a more understandable, visual way (see example 5.6).

The proposed solution is to split the family $\left\{h_{i}\right\}_{i \in I}$ into subfamilies $\left\{h_{i_{j}}\right\}_{i_{j} \in I_{j}}$ of compatible fuzzy subsets, each subfamily to be replaced afterwards by a quasi-arithmetic mean of all its members, that is, a new fuzzy subset $h_{j}^{\prime}$.

The key concept behind such grouping, splitting and further aggregating is the order induced by a fuzzy attribute on $X$. The structure of these orders is studied in Section 3. Section 4 deals with the simplest case, in which only one induced order is involved. Most of the results presented there are already known. Section 5 and 6 are new. Section 5 deals with the general case, and contains the main contributions of this paper. Section 6 makes use of the so-called betweenness relations [8] in order to explore the structure of the averaged similarities $\bar{E}$ when the fuzzy attributes $\left\{h_{i}\right\}_{i \in I}$ are unknown. 


\section{Preliminaries}

The majority of the results in this paper refer to $T$-indistinguishability operators with respect to a continuous Archimedean $t$-norm. Formally, a $t$-norm is Archimedean if and only if for each $\mathrm{x}, \mathrm{y}$ in the open interval $(0,1)$ there is a natural number $\mathrm{n}$ such that $x^{n} \leqslant y$, where $x^{n}=T(\ldots T(x, T(x, x)) \ldots)(n$ times).

Every continuous Archimedean $t$-norm is isomorphic to the sum of positive real numbers, bounded or unbounded, according to Ling's theorem [6]. The order reversing isomorphism $t:[0,1] \rightarrow[0,+\infty]$ is called an additive generator of $T$, and $T(a, b)=t^{[-1]}(t(a)+t(b))$ for all $a, b \in[0,1]$ where $t^{[-1]}$ is the pseudo-inverse of $t$.

The standard examples of continuous Archimedean $t$-norms are $E$ (the Eukasiewicz $t$-norm) and $P$, the product $t$-norm. The most prominent example of continuous $t$-norm which is not Archimedean is $T=M I N$.

If the $t$-norm $T$ has an additive generator $t$, the $T$-transitivity (definition 1.1.3) can be understood as a particular version of the triangle inequality for metrics, since $T(E(x, y), E(y, z)) \leq E(x, z)$ can be rewritten as $t(E(x, y))+t(E(y, z)) \geq t(E(x, z))$ or, in a more convenient notation for the purposes of this paper,

$$
(t \circ E)(x, y)+(t \circ E)(y, z) \geq(t \circ E)(x, z) \text {. }
$$

Such interpretation allows us to think of similar objects as close objects in a metric sense. Although $T=M I N$ lacks an additive generator, it behaves similarly to its Archimedean counterparts, only that it becomes associated to an ultrametric instead of a proper metric [9].

Each fuzzy subset $h_{i}: X \rightarrow[0,1], i \in I$ induces a similarity on $X$ by means of $E_{T}$, the biresiduation, or natural indistinguishability with respect to T on $[0,1]$.

Definition 2.1. The residuated implication of a t-norm $T$ is the map $I_{T}$ : $[0,1] \times[0,1] \rightarrow[0,1]$ defined by $I_{T}(a, b)=S U P\{\alpha \in[0,1] / T(\alpha, a) \leqslant b\}$, for all $a, b \in[0,1]$

Definition 2.2. The residuated biimplication, or natural indistinguishability, of a $t$-norm $T$ is the map $E_{T}:[0,1] \times[0,1] \rightarrow[0,1]$ defined by $E_{T}(a, b)=$ $M I N\left\{I_{T}(a, b), I_{T}(b, a)\right\}$ 
As the $t$-norm $T$ represents the multivalued connective $A N D$, the residuated implication $I_{T}$ and the natural indistinguishability $E_{T}$ associated to $T$ represent the multivalued implication and biimplication on the unit interval, respectively. Accordingly, they are often written as $\rightarrow_{T}$ and $\leftrightarrow_{T}$ respectively.

It is worth noticing that the natural indistinguishability $E_{T}$ is indeed a $T$-indistinguishability in the sense of definition 1.1.

For the purposes of this paper, the following lemmata provide operative definitions of $E_{T}$ in the case of a continuous Archimedean $t$-norm, or $T=$ $M I N$.

Lemma 2.3. Given a continuous Archimedean t-norm $T$, and $t$ an additive generator of $T$, the natural indistinguishability operator $E_{T}$ is the $T$-indistinguishability operator on the unit interval defined by $E_{T}(x, y)=$ $t^{[-1]}(|t(x)-t(y)|)$ for all $x, y \in[0,1]$.

Lemma 2.4. The natural indistinguishability $E_{M I N}$ is the MIN-indistinguishability operator on the unit interval defined for all $x, y \in[0,1]$ by

$$
E_{M I N}(x, y)= \begin{cases}1, & \text { if } x=y \\ M I N(x, y), & \text { otherwise }\end{cases}
$$

Each fuzzy subset $h_{i}: X \rightarrow[0,1]$ induces a $T$-indistinguishability operator on $X$ via $E_{h_{i}}(x, y)=E_{T}\left(h_{i}(x), h_{i}(y)\right)$, and the Representation Theorem states that every indistinguishability $E$ can be obtained from $T$-indistinguishability operators $E_{h_{i}}$ induced by single fuzzy attributes $h_{i}$.

Theorem 2.5. [10] Representation Theorem. Let $E$ be a fuzzy relation on a set $X$ and $T$ a continuous t-norm. $E$ is a T-indistinguishability operator if and only if there exists a family $H=\left\{h_{i}\right\}_{i \in I}$ of fuzzy subsets of $X$ such that for all $x, y \in X$

$$
E(x, y)=\inf _{i \in I} E_{h_{i}}(x, y)
$$

Definition 2.6. [1] The quasi-arithmetic mean $M$ in $[0,1]$ generated by a continuous strict monotonic map $t:[0,1] \rightarrow[-\infty, \infty]$ is defined for all $n \in \mathbb{N}$ and $x_{1}, \ldots, x_{n} \in[0,1]$ by

$$
M\left(x_{1}, \ldots x_{n}\right)=t^{-1}\left(\frac{t\left(x_{1}\right)+\ldots+t\left(x_{n}\right)}{n}\right) .
$$

$M$ is continuous if and only if Im $t \neq[-\infty, \infty]$, (where Im $t$ is the set of all $z$ such that $z=t(x)$ for some $x \in[0,1])$. 
Proposition 2.7. [9] The map assigning to every continuous Archimedean tnorm $T$ with additive generator $t$ the quasi-arithmetic mean $m_{t}$ generated by $t$ is a canonical bijection between the set of continuous Archimedean t-norms and continuous quasi-arithmetic means with $t(1) \neq \pm \infty$.

Similarly, weighted quasi-arithmetic means can be defined in the following way.

Definition 2.8. Let $\alpha_{1}, \alpha_{2}, \ldots, \alpha_{n}$ be positive numbers such that $\sum_{i=1}^{n} \alpha_{i}=$ 1. $\alpha_{i}$ are called weights. The weighted quasi-arithmetic mean $M^{\alpha_{1}, \alpha_{2}, \ldots, \alpha_{n}}$ of $x_{1}, x_{2}, \ldots, x_{n} \in[0,1]$ with weights $\alpha_{1}, \alpha_{2}, \ldots, \alpha_{n}$ generated by a continuous strict monotonic map $t:[0,1] \rightarrow[-\infty, \infty]$ is

$$
M^{\alpha_{1}, \alpha_{2}, \ldots, \alpha_{n}}\left(x_{1}, x_{2}, \ldots, x_{n}\right)=t^{-1}\left(\sum_{i=1}^{n} \alpha_{i} \cdot t\left(x_{i}\right)\right) .
$$

$M^{\alpha_{1}, \alpha_{2}, \ldots, \alpha_{n}}$ is continuous if and only if Im $t \neq[-\infty, \infty]$.

Proposition 2.9. [9] The map assigning to every continuous Archimedean tnorm $T$ with additive generator the weighted quasi-arithmetic mean $M^{\alpha_{1}, \alpha_{2}, \ldots, \alpha_{n}}$ generated by $t$ is a canonical bijection between the set of continuous Archimedean t-norms and continuous weighted quasi-arithmetic means with weights $\alpha_{1}, \alpha_{2}, \ldots, \alpha_{n}$ and with $t(1) \neq \pm \infty$.

For simplicity, we well write $M\left(\alpha_{i}, x_{i}\right)$ instead of $M^{\alpha_{1}, \alpha_{2}, \ldots, \alpha_{n}}\left(x_{1}, x_{2}, \ldots, x_{n}\right)$. The Łukasiewicz $t$-norm provides a specially simple example of quasiarithmetic mean.

Example 2.10. Consider the additive generator $t:[0,1] \rightarrow[0,1]$ defined by $t(a)=1-a$. Its quasi-inverse is

$$
t^{[-1]}(b)=\left\{\begin{array}{l}
0, \text { if } b \geqslant 1 \\
1-b, \text { if } 0 \leqslant b<1 \\
1, \text { if } b<0
\end{array}\right.
$$

The t-norm $T(a, b)=t^{[-1]}(t(a)+t(b))$ is then the Eukasiewicz $t$-norm, $T(a, b)=M A X(a+b-1,0)$, and the associated quasi-arithmetic mean is $M\left(\alpha_{i}, a_{i}\right)=\sum_{i=1}^{n} \alpha_{i} a_{i}=\sum_{i=1}^{n} \frac{1}{n} a_{i}$, the standard arithmetic mean, provided that $\alpha_{i}=\frac{1}{n}$ for all $i=1, \ldots, n$.

Similarly, it is easy to show that the quasi-arithmetic mean associated to the product $t$-norm is the standard geometric mean. 


\section{Orders Induced by Fuzzy Attributes}

In this section the structure of the orders induced by families of fuzzy subsets on a finite set $X$ is thoroughly explained. We represent fuzzy attributes, or criteria, by means of fuzzy subsets $h: X \rightarrow[0,1]$, and any fuzzy subset $h$ naturally induces the order of $[0,1]$ on $X$. We will note this order (which is, in general, a preorder) by $\leq_{h}$. Formally:

Definition 3.1. A relation on $X$ is called:

3.1.1 A preorder if it is reflexive and transitive.

3.1.2 An order if it is reflexive, transitive and antisymmetric.

3.1.3 A total preorder if it is reflexive, transitive and such that $x \leq y$ or $y \leq x$ for all $x, y \in X$.

Definition 3.2. Induced preorder. The preorder induced by a fuzzy subset $h: X \rightarrow[0,1]$ on $X, \leq_{h}$, is defined by $x \leq_{h} y$ if and only if $h(x) \leq h(y)$.

It is straightforward to check that $\leq_{h}$ is, indeed, a preorder on $X$, and a total one, since $[0,1]$ is a totally ordered set.

Lemma 3.3. For all fuzzy subsets $h: X \rightarrow[0,1]$, the relation $\leq_{h}$ is a total preorder on $X$.

Accordingly, a family of fuzzy subsets $H=\left\{h_{i}\right\}_{i \in I}$ induces a family of total preorders $\leq_{h_{i}}, i \in I$, on $X$, the structure of which is key to reduce the complexity of the similarities obtained as averages of the singly generated similarities $E_{h_{i}}, i \in I$.

NOTE: Informally, we will refer to such total preorders simply as the orders induced by $H$.

A set $X$ with a total order defined on it is called a totally ordered set. The most prominent example in our context is the unit interval $[0,1]$ with $\leq$, the standard real line's order.

Example 3.4. An important example of a total preorder which is not an order is provided by $X=[0,1]^{N}$. For a fixed $k=1 \ldots N$ we consider the relation $\leq_{k}$ defined by $x \leq_{k} y$ if, and only if $x_{k} \leq y_{k}$, where $x=\left(x_{1}, \ldots, x_{N}\right)$ and $y=\left(y_{1}, \ldots, y_{N}\right)$.

For a given preorder $\leq_{i}$, we will use $x<_{i} y$ to indicate the fact that $x \leq_{i} y$ holds while $y \leq_{i} x$ does not. A necessary, but not sufficient, condition for which is $x \neq y$. 
Similarly, we will use $x=_{i} y$ when $x \leq_{i} y$ and $y \leq_{i} x$. If $\leq_{i}$ is an order, then $x={ }_{i} y$ if and only if $x=y$.

Definition 3.5. [2] Two total preorders $\leq_{i}$ and $\leq_{j}$ on $X$ are compatible if and only if $x<_{i} y \Rightarrow x \leq_{j}$ y for all $x, y \in X$

If $\leq_{i}$ and $\leq_{j}$ on $X$ are compatible we will write $\leq_{i} \sim \leq_{j}$.

The notion of compatibility of orders is central to this paper. It is perhaps better grasped by saying that $x<_{i} y$ and $y<_{j} x$ cannot hold at the same time, provided that $\leq_{i} \sim \leq_{j}$. That is, the order $\leq_{i}$ cannot revert the order $\leq_{j}$

Proposition 3.6. Let $P(X)$ be the set of all total preorders on $X$. Then $\sim$ is a reflexive and symmetric relation on $P(X)$.

Proof. The relation is trivially reflexive.

As for symmetry, we have:

$\leqslant_{i} \sim \leqslant_{j}$ iff $(\forall x, y)$ if $x<_{i} y$ then $x \leqslant_{j} y$ iff

$(\forall x, y)$ if $\neg\left(x \leqslant_{j} y\right)$ then $\neg\left(x<_{i} y\right)$ iff

$(\forall x, y)$ if $y<_{j} x$ then $y \leqslant_{i} x$ iff $\leqslant_{j} \sim \leqslant_{i}$.

Proposition 3.7. Let $O(X)$ be the set of all total orders on $X$. The relation $\sim$ is the standard equality of orders on $O(X)$.

Proof. Given $\leq_{i}, \leq_{j} \in O(X)$, we want to see that if $\leq_{i} \sim \leq_{j}$ then $\leq_{i}$ and $\leq_{j}$ are the same order. That is, that if $\leq_{i} \sim \leq_{j}$ then, for all $x, y \in X, x \leq_{i} y$ if and only if $x \leq_{j} y$.

For all $x, y \in X$, if $x \leq_{i} y$ then $x=_{i} y$ or $x<_{i} y$. Since the order $\leq_{i}$ is total, $x={ }_{i} y$ is only possible when $x=y$. It follows that $x=y$ or $x \leq_{j} y$, or simply that $x \leq_{j} y$. We have proved that if $x \leq_{i} y$ then $x \leq_{j} y$ with independence of the indices $i$ and $j$, and therefore that $x \leq_{i} y$ if and only if $x \leq_{j} y$.

Although $\sim$ reduces to the standard equality of orders when restricted to $O(X)$, it becomes a more general kind of relation when defined on $P(X)$.

Example 3.8. Consider $X=\left\{x_{1}, x_{2}, x_{3}, x_{4}\right\}$ and the total preorders $\leq_{a}, \leq_{b}$ and $\leq_{c}$ defined by:

$$
x_{1}<_{a} x_{2}<_{a} x_{3}<{ }_{a} x_{4}
$$




$$
\begin{aligned}
& x_{1}<_{b} x_{2}=_{b} x_{3}<_{b} x_{4} \\
& x_{1}<_{c} x_{3}<_{c} x_{2}<_{c} x_{4}
\end{aligned}
$$

Then $\leqslant_{a} \sim \leqslant_{b}$ (that is, $\leq_{a}$ and $\leq_{b}$ are compatible), and $\leqslant_{b} \sim \leqslant_{c}$, but $\leq_{a} \nsim \leq_{c}$. In fact, when restricted to the set $P^{\prime}=\left\{\leq_{a}, \leq_{b}, \leq_{c}\right\} \subset P(X)$ we have that

$$
\begin{aligned}
& {\left[\leqslant_{a}\right]_{\sim}=\left\{\leqslant_{a}, \leqslant_{b}\right\}} \\
& {\left[\leqslant_{c}\right]_{\sim}=\left\{\leqslant_{b}, \leqslant_{c}\right\}} \\
& {\left[\leqslant_{b}\right]_{\sim}=\left\{\leqslant_{a}, \leqslant_{b}, \leqslant_{c}\right\}}
\end{aligned}
$$

where $\left[\leqslant_{i}\right]_{\sim}$ means the class of $\leqslant_{i}$ by the relation being compatible. Such classes do not constitute a partition.

NOTE: we use the term class in a broad sense, meaning by it the set of all the elements related to a particular one, even when the involved relation is not an equivalence.

Let us focus on the orders induced on $X$ by fuzzy attributes (Definition $3.2)$.

Proposition 3.9. Let $h: X \rightarrow[0,1]$ be a fuzzy subset, and let $\leq_{h}$ be the preorder induced on $X$ by $h$. Then $h$ is injective if and only if $\leq_{h}$ is a total order on $X$.

Proof. Trivial.

As a consequence, a given family of fuzzy subsets $H=\left\{h_{i}\right\}_{i \in I}$ inherits the notion of compatibility defined previously for total preorders.

Definition 3.10. Two fuzzy subsets $h, g: X \rightarrow[0,1]$ are compatible if their respectively induced preorders are compatible, $\leq_{h} \sim \leq_{g}$.

We will use $h \sim g$ to indicate the compatibility of $h$ and $g$.

Corollary 3.11. Let $H=\left\{h_{i}\right\}_{i \in I}$ be a family of fuzzy subsets. Then $\sim i s$ a reflexive and symmetric relation on $H$.

Proof. It is a consequence of Proposition 3.6.

Corollary 3.12. Let $H=\left\{h_{i}\right\}_{i \in I}$ be a family of fuzzy subsets. If $h_{i}$ is injective for all $i \in I$ then $\sim$ defines an equivalence relation on $H$. 
Proof. It is a consequence of Proposition 3.7, and the fact that injective fuzzy subsets induce total orders on $X$.

The injectivity of fuzzy subsets $h_{i}$ is not a necessary condition for $\sim$ to be an equivalence relation. As a rule of thumb, we may say that families $H$ with a small number of fuzzy subsets compared to the number of elements of $X$ tend to make of $\sim$ an equivalence relation. In general, carefully chosen families $H$ provide equivalence relations and, therefore, partitions of $H$.

Example 3.13. Consider $X=\left\{x_{1}, x_{2}, x_{3}, x_{4}, x_{5}, x_{6}\right\}$ and three fuzzy subsets $h_{a}, h_{b}, h_{c}: X \rightarrow[0,1]$ such that:

$$
\begin{aligned}
& h_{a}\left(x_{1}\right)<h_{a}\left(x_{2}\right)=h_{a}\left(x_{3}\right)<h_{a}\left(x_{4}\right)=h_{a}\left(x_{5}\right)<h_{a}\left(x_{6}\right) \\
& h_{b}\left(x_{1}\right)=h_{b}\left(x_{2}\right)<h_{b}\left(x_{3}\right)<h_{b}\left(x_{4}\right)<h_{b}\left(x_{5}\right)=h_{b}\left(x_{6}\right) \\
& h_{c}\left(x_{1}\right)=h_{c}\left(x_{2}\right)<h_{c}\left(x_{4}\right)<h_{c}\left(x_{3}\right)=h_{c}\left(x_{5}\right)<h_{c}\left(x_{6}\right)
\end{aligned}
$$

Then $h_{a} \sim h_{b}$ (that is, $h_{a}$ and $h_{b}$ are compatible), but $h_{a} \nsim h_{c}$ and $h_{b} \nsim h_{c}$. The set $H=\left\{h_{a}, h_{b}, h_{c}\right\}$ becomes therefore partitioned into two equivalence classes:

$$
\begin{aligned}
& {\left[h_{a}\right]_{\sim}=\left\{h_{a}, h_{b}\right\}} \\
& {\left[h_{b}\right]_{\sim}=\left\{h_{a}, h_{b}\right\}} \\
& {\left[h_{c}\right]_{\sim}=\left\{h_{c}\right\}}
\end{aligned}
$$

Another interesting feature of the injective fuzzy subsets is that they may be thought of as the prototypes at the core of the compatibility relation's classes, even if such classes are not proper ones and do not constitute a partition of $H$. If the compatibility relation $\sim$ defined on $H$ is not an equivalence relation, but each of its classes has at least one injective fuzzy subset, then the overlapping among classes are entirely made of non-injective fuzzy subsets.

Proposition 3.14. Let $f, g, h: X \rightarrow[0,1]$ be fuzzy subsets such that $f$ and $g$ are injective, and $f \nsim g$. If $h \sim f$ and $h \sim g$ then $h$ is non-injective.

Proof. Since $f \nsim g$ there exist $x, y \in X$ such that $f(x)<f(y)$ and $g(x)>$ $g(y)$, and because $h \sim f$ and $h \sim g$ then both $h(x) \leqslant h(y)$ and $h(x) \geqslant h(y)$ hold, and therefore $h(x)=h(y)$.

Also, if a class has at least one injective fuzzy subset, transitivity is guaranteed inside that particular class. 
Proposition 3.15. Let $f, g, h: X \rightarrow[0,1]$ be fuzzy subsets of $X$ such that $g \sim f$ and $h \sim f$. If $f$ is injective, then $g \sim h$.

Proof. For a given pair $x, y \in X$, if $h(x)<h(y)$ then $f(x)<f(y)$ ( for $h \sim f$ and $f$ is injective) and therefore $g(x) \leqslant g(y)$ (since $f \sim g$ ).

The preorders $\leq_{h_{i}}$ induced on $X$ by the fuzzy subsets $h_{i} \in H$ and the (classical) compatibility relation $\sim$ defined on them are key to the study of the (fuzzy) similarity relations because they explain how some subsets of fuzzy attributes $h_{i}$ may be merged into a single one $g$, depending on the compatibility of the induced preorders $\leq_{h_{i}}$.

In Section 4, the families $H$ are studied such that the compatibility relation $\sim$ is an equivalence relation whose associated partition reduces to a single class. Such families of fuzzy attributes can be merged into one single attribute. In Section 5, families $H$ yielding many equivalence classes or having non-transitive compatibility relations are considered.

\section{Averaged Indistinguishabilities that can be Generated by a Single Fuzzy Set}

In this section we consider families $H$ containing only compatible fuzzy attributes. The compatibility relation $\sim$ is then an equivalence relation having only one class. Thus, $[h]_{\sim}=H$ for all $h \in H$.

In [2] some results are given concerning such families $H$, showing that they can be replaced by a single fuzzy subset obtained as an arithmetic mean of all the fuzzy subsets in $H$. Let us recall some of them.

Let $T$ be a continuous Archimedean $t$-norm with additive generator $t$, and $M$ a quasi-arithmetic mean with the same generator $t$ as $T$, and weights $\alpha_{i}, i \in I$.

Given a family of fuzzy subsets $H=\left\{h_{i}\right\}_{i \in I}$, let us note $\bar{E}=M\left(\alpha_{i}, E_{h_{i}}\right)$, that is, $\bar{E}$ represents the quasi-arithmetic mean of the $T$-indistinguishability operators $E_{h_{i}}$. On the other hand, we may consider the singly generated $T$ indistinguishability operator $E_{\bar{h}}$ where $\bar{h}: X \rightarrow[0,1]$ is the quasi-arithmetic mean of the $h_{i}$ 's, that is, $\bar{h}=M\left(\alpha_{i}, h_{i}\right)$. In this situation, the following propositions hold.

Proposition 4.1. $E_{\bar{h}}$ is an indistinguishability operator with respect to $T$. 
Proof. Obvious, since $E_{\bar{h}}$ is the $T$-indistinguishability generated by the fuzzy subset $\bar{h}$.

Proposition 4.2. [7] [9] $\bar{E}$ is an indistinguishability operator with respect to $T$.

Proposition 4.3. [7] $\bar{E} \leq E_{\bar{h}}$

Proposition 4.4. [2] $\bar{E}=E_{\bar{h}}$ if, and only if, $h_{i} \sim h_{j}$ for all $i, j \in I$.

Proposition 4.4 is the key result upon which Section 5 is built. If a set of fuzzy criteria does not constitute a single class with respect to the compatibility relation $\sim$, it can still be split into as many subsets as classes, and for each subset a single fuzzy set is enough to generate the relation.

Slightly different results are obtained when the $t$-norm $T=M I N$ is considered.

Proposition 4.5. If $T=M I N, E_{\bar{h}}$ is an indistinguishability operator with respect to the MIN t-norm.

Proof. See proof 4.1.

Proposition 4.6. [2] Let $T=M I N$. If for all $x, y \in X$ either $h_{i}(x)<h_{i}(y)$ for all $i \in I$, or $h_{i}(y)<h_{i}(x)$ for all $i \in I$, then $\bar{E}=E_{\bar{h}}$.

It is worth noting that the previous proposition holds for any considered quasi-arithmetic mean, even though there is no possible concordance between the additive generator $t$ of the mean $M$ and that of the $t$-norm, since $T=$ $M I N$ lacks one.

Another interesting consequence is that although $\bar{E}$ is not, in general, a $M I N$-transitive fuzzy relation, it is so if the conditions of Proposition 4.6 hold, because $\bar{E}=E_{\bar{h}}$, and $E_{\bar{h}}$ is a $M I N$-indistinguishability operator, according to Proposition 4.5.

Corollary 4.7. In the conditions of Proposition 4.6, $\bar{E}$ is a MIN-indistinguishability operator.

Next lemma shows that conditions of Proposition 4.6 hold in case only injective fuzzy subsets are considered.

Lemma 4.8. If $h_{i} \sim h_{j}$ for all $i, j \in I$ and $h_{i}$ is injective for all $i \in I$, then for all $x, y \in I$ either $h_{i}(x)<h_{i}(y)$ for all $i \in I$ holds, or $h_{i}(y)<h_{i}(x)$ for all $i \in I$ does. 
Proof. Compatibility of $h_{i}$ and $h_{j}$ reduces to $h_{i}(x)<h_{i}(y) \Rightarrow h_{j}(x)<h_{j}(y)$ if only injective fuzzy subsets $h_{i}$ and $h_{j}$ are considered.

Therefore, a unified result for both the Archimedean and the MIN case can be provided in the injective case.

Proposition 4.9. Let $T$ be a continuous Archimedean $t$-norm additively generated by $t$, and $M$ a quasi-arithmetic mean with the same generator $t$. For both $T$ and the $t$-norm $M I N$, if $h_{i} \sim h_{j}$ for all $i, j \in I$, and $h_{i}$ is injective for all $i \in I$, then $\bar{E}_{H}=E_{\bar{h}}$

\section{The General Case: Reduction of the Num- ber of Attributes}

In this section we assume no restrictive conditions on the orders induced by the fuzzy subsets $H=\left\{h_{i}\right\}_{i \in I}$ on the finite set $X$. The goal is to split the family $H$ into subfamilies $H_{j}$ made of compatible attributes that can be merged into a single one $g_{j}$. The smaller the number of subfamilies $H_{j}$, the simpler the representation.

It is worth noticing that the fuzzy subsets $g_{j}$ do not necessarily belong to the the original family $H=\left\{h_{i}\right\}_{i \in I}$, because they are built as quasiarithmetic means of elements of $H$. A new family $G=\left\{g_{j}\right\}_{j \in J}$ with (in general) $g_{j} \neq h_{i}$, and having fewer elements than $H$, is therefore obtained.

As in Section 4, we shall deal first with continuous Archimedean $t$-norms, and shall consider $T=M I N$ afterwards.

Let $H=\left\{h_{i}\right\}_{i \in I}$ be a family of fuzzy subsets of $X$ which generates an averaged similarity $\bar{E}=\underset{i \in I}{M}\left(\alpha_{i}, E_{h_{i}}\right)$.

We first consider the case when the family $H=\left\{h_{i}\right\}_{i \in I}$ has been previously split into disjoint subsets of compatible elements. Formally, we assume that there is a family of subsets $\left\{H_{j}\right\}_{j \in J}$ of $H$ such that $H=\bigcup_{j \in J} H_{j}$ and $H_{j} \cap H_{j^{\prime}}=\emptyset$ if $j \neq j^{\prime}$, a compact notation for which is $H=\bigcup_{j \in J}^{\circ} H_{j}$, and such that $h_{k} \sim h_{k^{\prime}}$ for any arbitrarily chosen $h_{k}, h_{k^{\prime}} \in H_{j}$.

Note that, in such situation, also the family of indexes $I$ becomes partitioned into disjoint subsets, $I=\bigcup_{j \in J}^{\circ} I_{j}$ so that $H_{j}=\left\{h_{k}\right\}_{k \in I_{j}}$ for all $j \in J$. 
Obviously, the set $J$ has less or, at most, the same number of elements than the set $I,|J| \leqslant|I|$. Thus it is justified to talk about reduction of attributes, meaning a reduction in their numbers.

Theorem 5.1. Let $T$ be an Archimedean continuous t-norm and $M$ a quasiarithmetic mean with weights $\left(\alpha_{i}\right)_{i \in I}$ and the same additive generator as $T$. Let $H=\left\{h_{i}\right\}_{i \in I}$ be a family of fuzzy subsets of $X$, such that $H=\bigcup_{j \in J}^{\circ} H_{j}$, and that $h_{k} \sim h_{k^{\prime}}$ for all $k, k^{\prime} \in I_{j}$. Then there exists a family $G=\left\{g_{j}\right\}_{j \in J}$ of fuzzy subsets of $X$ and weights $\left(\beta_{j}\right)_{j \in J}$ such that $\bar{E}=M_{i \in I}\left(\alpha_{i} E_{h_{i}}\right)=$ $M_{j \in J}\left(\beta_{j}, E_{g_{j}}\right)$.

Proof. 5.1. Our goal is to build a family of fuzzy subsets $\left(g_{j}\right)_{j \in J}$ and weights $\left(\beta_{j}\right)_{j \in J}$ such that $\bar{E}=\underset{i \in I}{M}\left(\alpha_{i}, E_{h_{i}}\right)=\underset{j \in J}{M}\left(\beta_{j}, E_{g_{j}}\right)$. Each fuzzy set $g_{j}$ will be obtained as the quasi-arithmetic mean of all the fuzzy subsets in the subset $H_{j}$, that is, $g_{j}=\bar{h}_{j}=\underset{k \in I_{j}}{M}\left(\gamma_{k}, E_{h_{k}}\right)$ for some wheights $\gamma_{k}$ which will be naturally inferred from the following equalities.

$$
\begin{aligned}
& \bar{E}(x, y)=M_{i \in I}\left(\alpha_{i}, E_{h_{i}}(x, y)\right)=t^{[-1]}\left(\sum_{i \in I} \alpha_{i}\left(t \circ E_{h_{i}}\right)(x, y)\right) \\
& =t^{[-1]}\left(\sum_{j \in J}\left(\sum_{k \in I_{j}} \alpha_{k}\left(t \circ E_{h_{k}}\right)(x, y)\right)\right) \\
& \underset{(1)}{=t^{[-1]}}\left(\sum_{j \in J} \beta_{j}\left(\sum_{k \in I_{j}} \frac{\alpha_{k}}{\beta_{j}}\left(t \circ E_{h_{k}}\right)(x, y)\right)\right) \\
& =t^{[-1]}\left(\sum_{j \in J} \beta_{j}\left(t \circ t^{[-1]}\right)\left(\sum_{k \in I_{j}} \frac{\alpha_{k}}{\beta_{j}} t \circ E_{h_{k}}(x, y)\right)\right) \\
& \underset{(2)}{=} t^{[-1]}\left(\sum_{j \in J} \beta_{j} t\left(\underset{k \in I_{j}}{M}\left(\gamma_{k}, E_{h_{k}}(x, y)\right)\right)\right) \\
& \underset{(3)}{=} t^{[-1]}\left(\sum_{j \in J} \beta_{j}\left(t \circ E_{g_{j}}\right)(x, y)\right) \underset{(4)}{=} \underset{j \in J}{M}\left(\beta_{j}, E_{g_{j}}\right)
\end{aligned}
$$


where the numbered equalities are consequence of:

(1) Define $\beta_{j}=\sum_{k \in I_{j}} \alpha_{k}$

(2) Define $\gamma_{k}=\frac{\alpha_{k}}{\beta_{j}}$ and note that $\sum_{k \in I_{j}} \gamma_{k}=1$

(3) Take $g_{j}=\bar{h}_{j}=\underset{k \in I_{j}}{M}\left(\gamma_{k}, E_{h_{k}}\right)$ and apply proposition 4.4 .

(4) Note that $\sum_{j \in J} \beta_{j}=1$

Corollary 5.2. In the conditions of the previous theorem, if at least one subset $H_{j}$ has more than one element, then $|J|<|I|$.

The previous theorem is built on the hypothesis that the family $H$ may be partitioned into disjoint subsets $H_{j}$ made up of compatible fuzzy subsets.

If the compatibility relation $\sim$ is transitive, and therefore an equivalence relation, then the set $H$ becomes naturally partitioned into its equivalence classes. Then each subset $H_{j}$ can be thought of as the class of any of its elements $h_{k}$, that is, $H_{j}=\left[h_{k}\right]_{\sim}$.

According to corollary 3.12 if all the $h_{i}$ are injective, then the compatibility relation $\sim$ defined on $H=\left\{h_{i}\right\}_{i \in I}$ is an equivalence relation, and therefore injective families of fuzzy subsets become naturally split into classes.

A similar theorem to 5.1 may be stated for $T=M I N$ if $H=\left\{h_{i}\right\}_{i \in I}$ is a family of injective fuzzy sets.

Theorem 5.3. Let $M$ be a quasi-arithmetic mean with weights $\left(\alpha_{i}\right)_{i \in I}, T=$ $M I N$, and $H=\left\{h_{i}\right\}_{i \in I}$ a family of injective fuzzy subsets of $X$. Then $\bar{E}=M_{i \in I}\left(\alpha_{i}, E_{h_{i}}\right)=M_{j \in J}\left(\beta_{j}, E_{g_{j}}\right)$ for some family $G=\left\{g_{j}\right\}_{j \in J}$ of fuzzy subsets of $X$ and weights $\left(\beta_{i}\right)_{i \in I}$, where $\left\{\left[h_{j}\right]_{\sim}\right\}_{j \in J}$ is the set of all the $\sim$ equivalence classes.

Proof. The proof of 5.1 may be simply rewritten here, since $\left(\left[h_{j}\right]_{\sim}\right)_{j \in J}$ provides a partition of $H$, and equalitiy (3) holds for $T=M I N$ under the hypothesis of injectivity, according to 3.12 . 
Transitivity of the compatibility relation $\sim$ is not, though, a necessary condition. Indeed, we will provide a constructive proof of the fact that all families $H=\left\{h_{i}\right\}_{i \in I}$ can be split into disjoint subfamilies $\left\{H_{j}\right\}_{j \in J}$. Each $H_{j}$ is built upon an injective fuzzy subset $f_{j}$ which may or may not belong to $H$.

Proposition 5.4. Let $H=\left\{h_{i}\right\}_{i \in I}$ be a family of fuzzy subsets of $X$. Then, there exists a family $F=\left\{f_{j}\right\}_{j \in J}$ of injective fuzzy subsets of $X$ and, for each $f_{j}$ a subset $H_{j} \subseteq H$ such that $H=\bigcup_{j \in J}^{0} H_{j}$, and that $h_{k} \sim h_{k^{\prime}}$ for all $k, k^{\prime} \in I_{j}$.

Next lemma will be helpful in order to prove the previous proposition.

Lemma 5.5. Let $h: X \rightarrow[0,1]$ be a non-injective fuzzy subset of $X$. Then there exists an injective fuzzy subset $f: X \rightarrow[0,1]$ such that $h \sim f$.

Proof. Let us note $h\left(x_{i}\right)=a_{i}$, and suppose $a_{i}<a_{j}$ if $i<j$. Since $h$ is non-injective, $h(X)=\left\{a_{1}, \ldots, a_{k}\right\}$ with $k<n$.

If $k=1$ then any injective fuzzy set $f$ satisfies $h \sim f$.

Let us suppose $1<k$, and consider $b_{1}, \ldots, b_{k+1} \in[0,1]$ as follows:

$$
\begin{aligned}
& b_{1}=a_{1} \\
& b_{j}=\frac{a_{j}+a_{j-1}}{2} \text { if } j=2, \ldots, k \\
& b_{k+1}=a_{k}
\end{aligned}
$$

Then, if $A_{j}=h^{-1}\left(a_{j}\right)=\left\{x_{k_{1}}, \ldots, x_{k_{j}}\right\}$ we may consider any injective map $f_{j}: A_{j} \rightarrow\left(b_{j}, b_{j+1}\right)$ and the map $f: X \rightarrow[0,1]$ defined piecewise by $f\left(x_{i}\right)=f_{j}\left(x_{i}\right)$ if $x_{i} \in A_{j}$, is injective and compatible with $h$.

Let us proceed with the proof of proposition 5.4.

Proof. Let $F=\left\{f_{l}\right\}_{l \in L}$, the set of all injective fuzzy subsets of $X$. According to corollary 3.12, the compatibility relation $\sim$ defines an equivalence relation on $F$. Let $\left\{F_{m}\right\}_{m \in M}$ be the set of its classes, that is, $F_{m}=\left[f_{m}\right]_{\sim}$, for arbitrarily chosen $f_{m} \in F_{m}$. We then have that $F=\bigcup_{m \in M}^{\circ} F_{m}$.

We can construct a map $\psi: I \rightarrow M$ such that:

a. If $\psi(i)=m$ then $h_{i} \sim f_{m}$. 
b. If $\psi(i)=\psi(j)$ then $h_{i} \sim h_{j}$.

Indeed, for each $i \in I$ we consider an injective fuzzy set $f$ defined as in lemma 5.5, and define $\psi(i)=m$ if $f \in F_{m}$. Such a $\psi$ is well defined because $f$ belongs only to one equivalence class $F_{m}$. Defined in this way, obviously $h_{i} \sim f_{m}$. Also, for any other $k \in I$ such that $\psi(i)=\psi(k)$ proposition 3.15 assures that $h_{k} \sim h_{i}$.

Finally, the sets $H_{j}$ are obtained by taking $J=\operatorname{Im}(\psi), I_{j}=\psi^{-1}(j)$, for all $j \in J$. Construction ensures both that $I_{j} \cap I_{j^{\prime}}=\emptyset$ if $j \neq j^{\prime}$ and that $\bigcup_{j \in J} I_{j}=I$.

Note that the partition $\left\{H_{j}\right\}_{j \in J}$ obtained in the previous proof is, in general, not unique. Unless $\sim$ defines an equivalence relation on $H$, some non-injective fuzzy subsets $h_{i}$ may be assigned to different injective ones $f_{j}$ by lemma 5.5, and therefore to different classes $H_{j}$.

An interesting application that involves non-injective fuzzy subsets is shown in the next example, where a family of binary crisp attributes is packed into fewer fuzzy attributes. It shows how fuzzy concepts may be built from crisp ones by grouping those when the induced orders are taken into account.

Example 5.6. Synthesizing crisp data into fuzzy attributes.

Consider $X=\left\{x_{1}, x_{2}, x_{3}, x_{4}, x_{5}, x_{6}\right\}$ and a family of crisp binary attributes $H=\left\{h_{1}, h_{2}, h_{3}, h_{4}, h_{5}\right\}$ explicitly defined by the following matrix, where row $i$ represents the element $x_{i}$, and column $j$ represents the attribute $h_{j}$.

$\begin{array}{cccccc}x_{1} & 0 & 0 & 0 & 0 & 0 \\ x_{2} & 1 & 0 & 0 & 1 & 0 \\ x_{3} & 1 & 1 & 0 & 1 & 1 \\ x_{4} & 1 & 1 & 0 & 1 & 0 \\ x_{5} & 1 & 1 & 0 & 0 & 0 \\ x_{6} & 1 & 1 & 1 & 0 & 0 \\ & h_{1} & h_{2} & h_{3} & h_{4} & h_{5}\end{array}$

We define a similarity on $X$ by averaging the crisp equivalence relations $E_{h_{k}}\left(x_{i}, x_{j}\right)=1-\left|h_{k}\left(x_{i}\right)-h_{k}\left(x_{j}\right)\right|, k=1, \ldots, 5$, as $E=\sum_{k=1}^{5} \frac{1}{5} E_{h_{k}}$.

$E$ is an indistinguishability operator with respect to the Eukasiewicz tnorm, $T=E$. 
The preorders induced on $X$ by the attributes $h_{j}$ is as follows:

$$
\begin{aligned}
& x_{1}<_{h_{1}} x_{2}={ }_{h_{1}} x_{3}={ }_{h_{1}} x_{4}={ }_{h_{1}} x_{5}={ }_{h_{1}} x_{6} \\
& x_{1}={ }_{h_{2}} x_{2}<_{h_{2}} x_{3}={ }_{h_{2}} x_{4}={ }_{h_{2}} x_{5}={ }_{h_{2}} x_{6} \\
& x_{1}={ }_{h_{3}} x_{2}={ }_{h_{3}} x_{3}={ }_{h_{3}} x_{4}={ }_{h_{3}} x_{5}<_{h_{3}} x_{6} \\
& x_{1}={ }_{h_{4}} x_{5}={ }_{h_{4}} x_{6}<_{h_{4}} x_{2}={ }_{h_{4}} x_{3}={ }_{h_{4}} x_{4} \\
& x_{1}={ }_{h_{5}} x_{5}={ }_{h_{5}} x_{6}={ }_{h_{5}} x_{2}={ }_{h_{5}} x_{4}<_{h_{5}} x_{3}
\end{aligned}
$$

Without explicitely constructing the injective fuzzy subsets of Lemma 5.5, it is clear that the total order

$$
x_{1}<_{a} x_{2}<{ }_{a} x_{3}<_{a} x_{4}<{ }_{a} x_{5}<_{a} x_{6}
$$

is compatible with the preorders induced by $h_{1}, h_{2}$ and $h_{3}$, while the total order

$$
x_{1}<_{b} x_{5}<_{b} x_{6}<_{b} x_{2}<_{b} x_{4}<_{b} x_{3}
$$

is compatible with the preorders induced by $h_{4}, h_{5}$.

By averaging $h_{1}, h_{2}, h_{3}$ into $g_{1}$ and $h_{4}, h_{5}$ into $g_{2}$ the following matrix is obtained:

$$
\begin{array}{ccc}
x_{1} & 0 & 0 \\
x_{2} & 1 / 3 & 1 / 2 \\
x_{3} & 2 / 3 & 1 \\
x_{4} & 2 / 3 & 1 / 2 \\
x_{5} & 2 / 3 & 0 \\
x_{6} & 1 & 0 \\
& g_{1} & g_{2}
\end{array}
$$

The fuzzy relation $E$ may now be calculated as $E=\frac{3}{5} E_{g_{1}}+\frac{2}{5} E_{g_{2}}$

The next example shows how the previous constructs should be applied when dealing with real problems. The data used does not come from any specific experimental setting, but is generated in a realistic way nonetheless. We consider two types of attributes. First, continuous variables spreading over an interval, such as temperature, pressure, weight, height and so on. And second, variables tending to group around some specific values acting as prototypes, such as colour in hand stained glass tiles measured at the end of a production line for quality control purposes.

The reasons for taking several readings of each variable may vary depending on the nature of the attribute. For example, filtering a noisy continuous 
signal of the aforementioned first type, or measuring colour at many points of the same tile, as an example of the second type.

The process of attribute reduction tends to group the many readings of a variable into a single averaged variable. Allowing for some level of error inherent to every experimental setting, the failing to achieve that indicates that the noise is too high for a measured attribute to be considered a single variable, or that the assumption that some readings are the result of a single variable may in fact constitute an oversimplification of a much more complex situation.

On the other hand, an interesting feature of the attribute reducing process is the discovery of monotonic related attributes, such as size and weight or pressure and temperature, which are then synthesized into a single averaged attribute.

Example 5.7. Building a simplified similarity model in an experimental setting.

Three variables $v_{1}, v_{2}$ and $v_{3}$ are measured against the $n$ elements of a set $X$. We take $n_{1}$ readings of $v_{1}, n_{2}$ of $v_{2}$ and $n_{3}$ of $v_{3}$ and remap them into the unit interval. The normalized $j$-th reading of variable $v_{i}$ is noted by $v_{i j}$ (see matrix below). The first two variables are assumed to come from continuously spread attributes, while the third tends to cluster around some specific values. To simulate a real environment, Gaussian noise is added to the first two variables, and uniformly distributed noise to the third.

For displaying purposes, we run the simulation for $n_{1}=2, n_{2}=2, n_{3}=3$ and $n=8$. After that, we provide results for larger values of the parameters. Standard mathematical software such as Matlab, run on a conventional laptop, allows for values of $n$ up to thousands, provided that the number of measurements is kept reasonably low.

STEP 1. Data acquisition and normalisation.

After reordering according to the first column, the following matrix is obtained. Columns $h_{k}$ represent normalized readings of variables $v_{i j}$ and rows represent elements $x_{n}$. 


$\begin{array}{cccccccc} & v_{11} & v_{12} & v_{21} & v_{22} & v_{31} & v_{32} & v_{33} \\ x_{1} & 0.7008 & 0.6870 & 0.0946 & 0 & 0.7015 & 0.7473 & 0.7718 \\ x_{2} & 0.7586 & 0.7368 & 0.0189 & 0.2021 & 0.7664 & 0.7903 & 0.7569 \\ x_{3} & 0.7824 & 0.7708 & 0.2479 & 0.2343 & 0.7724 & 0.7451 & 0.7461 \\ x_{4} & 0.8365 & 0.8342 & 0.2956 & 0.3801 & 0.1282 & 0.1805 & 0.1445 \\ x_{5} & 0.8636 & 0.8687 & 0.5247 & 0.4754 & 0.1262 & 0.1829 & 0.1088 \\ x_{6} & 0.9017 & 0.9013 & 0.6449 & 0.5131 & 0.1708 & 0.1166 & 0.1443 \\ x_{7} & 0.9638 & 0.9483 & 0.7110 & 0.7435 & 0.5784 & 0.5394 & 0.5366 \\ x_{8} & 0.9861 & 0.9901 & 0.7924 & 0.8180 & 0.5986 & 0.5521 & 0.5303 \\ & h_{1} & h_{2} & h_{3} & h_{4} & h_{5} & h_{6} & h_{7}\end{array}$

STEP 2. Order matrix.

We compute a matrix displaying the permutations of each column against the first one.

$\begin{array}{cccccccc}x_{1} & 1 & 1 & 2 & 1 & 6 & 7 & 8 \\ x_{2} & 2 & 2 & 1 & 2 & 7 & 8 & 7 \\ x_{3} & 3 & 3 & 3 & 3 & 8 & 6 & 6 \\ x_{4} & 4 & 4 & 4 & 4 & 2 & 2 & 3 \\ x_{5} & 5 & 5 & 5 & 5 & 1 & 3 & 1 \\ x_{6} & 6 & 6 & 6 & 6 & 3 & 1 & 2 \\ x_{7} & 7 & 7 & 7 & 7 & 4 & 4 & 5 \\ x_{8} & 8 & 8 & 8 & 8 & 5 & 5 & 4 \\ & h_{1} & h_{2} & h_{3} & h_{4} & h_{5} & h_{6} & h_{7}\end{array}$

Note that columns $h_{1}$ to $h_{4}$ are ordered in almost the same way, despite the fact that the entries for columns $h_{1}$ and $h_{2}$ (variable $v_{1}$ ) and those for $h_{3}$ and $h_{4}$ (variable $v_{2}$ ) are quite different in value. This shows both sound measuring and monotonic dependence, or correlation, between $v_{1}$ and $v_{2}$. Variable $v_{3}$ (columns $h_{5}, h_{6}, h_{7}$ ) behaves independently of the other two, and suffers from higher levels of noise, reflected in the changing permutation patterns through columns.

STEP3. Reduction of attributes.

At a glance it becomes apparent that columns $h_{1}$ to $h_{4}$ may be averaged into one single attribute $g_{1}$, although not in an exact manner owing to column $h_{3}$. On the other hand, we may force columns $h_{5}$ to $h_{7}$ to become a new attribute $g_{2}$ despite the fact that their permutations differ in a more apparent way. The two new attributes, assuming equal weights i.e. $\alpha_{1}=\alpha_{2}=\alpha_{3}=\alpha_{4}=0.25$ for $h_{1}, h_{2}, h_{3}$, and $h_{4}$, and $\alpha_{1}=\alpha_{2}=\alpha_{3}=0.33$ for $h_{5}, h_{6}$ and $h_{7}$, are: 


$$
\begin{array}{ccccccccc}
g_{1} & 0.3706 & 0.4291 & 0.5089 & 0.5866 & 0.6831 & 0.7403 & 0.8417 & 0.8966 \\
g_{2} & 0.7402 & 0.7712 & 0.7545 & 0.1510 & 0.1393 & 0.1439 & 0.5515 & 0.5603 \\
& x_{1} & x_{2} & x_{3} & x_{4} & x_{5} & x_{6} & x_{7} & x_{8}
\end{array}
$$

STEP 4. Computing the errors.

We generate the original similarity $S$ by using the 7 attributes, and the similarity $T$ obtained from the 2 new attributes, with weights $\alpha_{1}=\frac{4}{7}, \alpha_{2}=\frac{3}{7}$. Then compute the normalized difference, or error, between the two by means of $e=\frac{1}{n^{2}} \sum_{i, j}\left|S\left(x_{i}, x_{j}\right)-T\left(x_{i}, x_{j}\right)\right|$, which yields $e=0.0022$.

\section{FILTERING.}

Optionally, we may relax precision by rounding the measured variables with respect to some specific discretized scale. This is a very simple digital filter, and some more sophisticated ones could be tried. Behind such approach stands the idea that some amount of disorder may be attributed to noise, and therefore corrected by filtering.

With this new set of data, we repeat all the 4 previous steps, and we obtain:

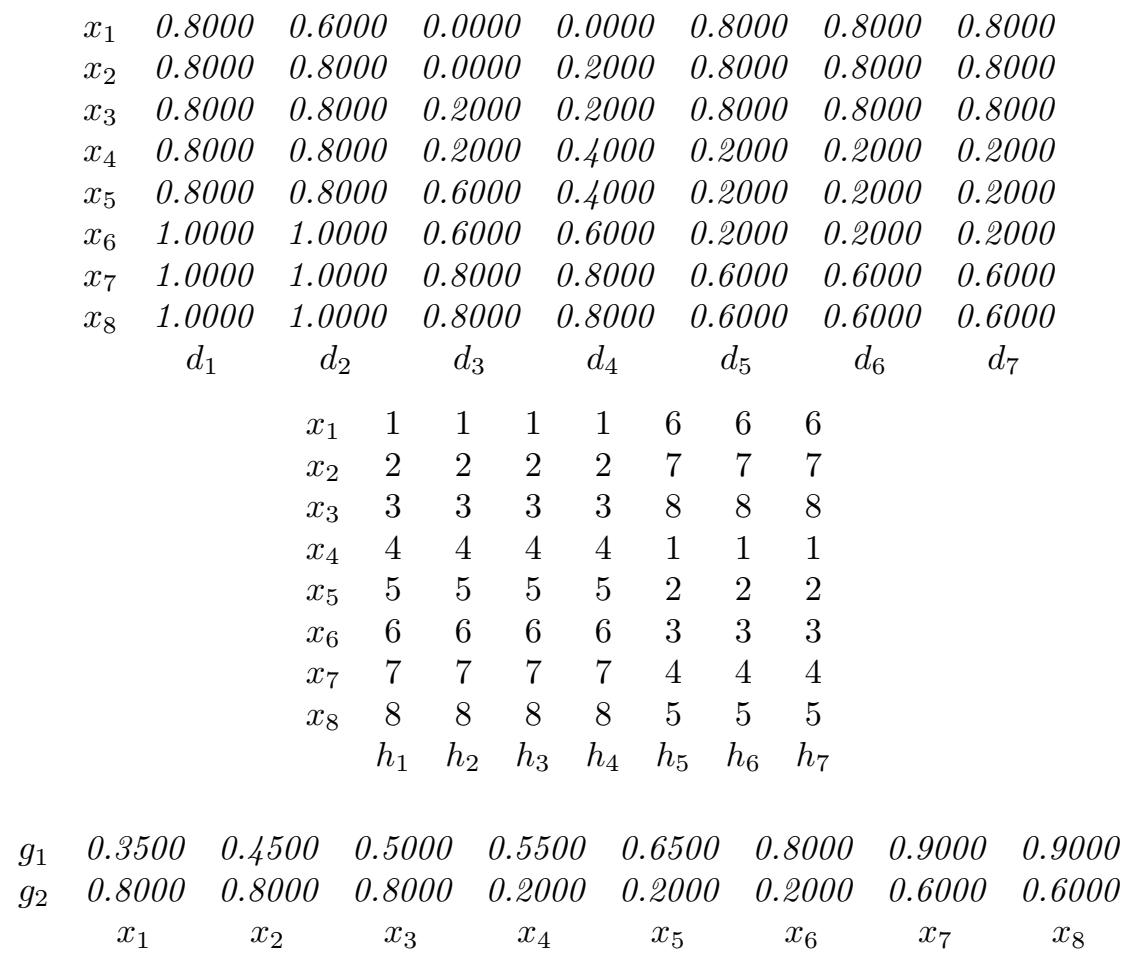


The error in this case becomes $e=0$.

\section{SIMULATION RESULTS}

Next table shows some results for bigger sets of data. They refer to the same set of variables $v_{1}, v_{2}$ and $v_{3}$. Each variable is measured 5 times, so that $n 1=n 2=n 3=5$ making the real number of variables amount to 15 . $X$ is a set of $n=1000$ elements. The levels of noise in each variable are designated by $e_{1}, e_{2}$ and $e_{3}$ respectively, and they represent standard deviations. No filtering is applied.

$\begin{array}{cccc}e 1 & e 2 & e 3 & e \\ 0.01 & 0.01 & 0.05 & 0.0013 \\ 0.02 & 0.02 & 0.1 & 0.0037 \\ 0.05 & 0.05 & 0.25 & 0.0157 \\ 0.1 & 0.1 & 0.25 & 0.0367\end{array}$

Finally, as a summary of the section, we provide a procedure to find a smaller set of attributes starting from a given one.

PROCEDURE: THE INJECTIVE CASE

If the family $H=\left\{h_{i}\right\}_{i \in I}$ consists only of injective fuzzy sets, then the relation $\sim$ is an equivalence relation on $H$. The procedure is valid for both $T$ continuous Archimedean and $T=M I N$, and can be stated as follows.

Start by chossing any fuzzy subset $h_{i_{1}} \in H$, and consider its equivalence class $H_{1}=\left\{h \in H\right.$ s.t.h $\left.\sim h i_{1}\right\}$.

If $H=H_{1}$, stop. Else, choose $h_{i_{2}} \in H-H_{1}$, and consider its equivalence class $H_{2}=\left\{h \in H\right.$ s.t. $\left.h \sim h i_{2}\right\}$.

If $H=H_{1} \stackrel{\circ}{\cup} H_{2}$, stop. Else, choose $h_{i_{3}} \in H-H_{1} \cup H_{2}$, and repeat until $H=\bigcup_{j \in J}^{\circ} H_{j}$.

Finally, for each subset $H_{j}$ we compute the new fuzzy attribute $g_{j}$ as the quasi-arithmetic mean of all the fuzzy subsets $h \in H_{j}$, that is, $g_{j}=\bar{h}_{j}=$ $\underset{h_{k} \in H_{j}}{M}\left(\gamma_{k}, h_{k}\right)$. The weights $\gamma_{k}$ are those in the proof of theorem 5.1.

THE GENERAL CASE

If the family $H=\left\{h_{i}\right\}_{i \in I}$ has some non injective fuzzy sets, then $\sim$ is not necessarily an equivalence relation on $H$. The procedure is valid only for continuous Archimedean t-norms, and consists in a simple modification of the previous one. 
At step $m$, if $H=\bigcup_{j=1 \ldots m}^{\circ} H_{j}$, stop. Else, choose $h_{i_{m+1}} \in H-\bigcup_{j=1 \ldots m}^{\circ} H_{j}$, construct an injective fuzzy subset $f_{i_{m+1}}$ according to lemma 5.5, and consider $H_{m+1}=\left\{h \in H\right.$ s.t.h $\left.\sim f_{i_{m+1}}\right\}$. Note that, in general, $f_{i_{m+1}} \notin H_{m+1}$, and it is only used to build $H_{m+1}$.

\section{Betweennes Relations and Averaged Simi- larities}

So far we have assumed that the fuzzy criteria $h_{i}$ used to generate an averaged similarity $\bar{E}=\underset{i \in I}{M}\left(\alpha_{i}, E_{h_{i}}\right)$ are explicitly known. In a practical setting, though, this is not always the case. A fuzzy relation may come from measurements directly related to the similarity between objects rather than to the values of a set of attributes. This is very often the case when we intend to build a similarity relation by questioning humans about their impressions and feelings.

If we are presented with two averaged similarities $\bar{E}=M_{i \in I}\left(\alpha_{i}, E_{h_{i}}\right)$ and $\bar{G}=\underset{k \in K}{M}\left(\beta_{k}, E_{g_{k}}\right)$ with unknown fuzzy subsets $h_{i}, g_{k}$ and weights $\alpha_{i}, \beta_{k}$, it will be interesting to know if some quasi-arithmetic mean $\bar{F}$ of $\bar{E}$ and $\bar{G}$, $\bar{F}=M(\bar{E}, \bar{G})$, can be simply expressed with the family of fuzzy subsets $\left\{h_{i}\right\}_{i \in I}$, or if the underlying fuzzy criteria $\left\{g_{k}\right\}_{k \in K}$ which determine $\bar{G}$ are compatible with those of $\bar{E}$.

Corollary 6.8 provides necessary conditions for the compatibility of such (unknown) families $\left\{h_{i}\right\}_{i \in I}$ and $\left\{g_{k}\right\}_{k \in K}$ which are based on the notion of betweenness, a ternary relation on $X$ that may be derived directly from the similarity $\bar{E}$ with no reference to the fuzzy subsets $h_{i}$. Whether similar sufficient conditions hold remains an open problem.

Definition 6.1. [8] A betweenness relation on a set $X$ is a ternary relation $B$ on $X$ such that for all $x, y, z \in X$ satisfies:

1. $(x, y, z) \in B \Rightarrow x \neq y \neq z \neq x$.

2. $(x, y, z) \in B \Rightarrow(z, y, x) \in B$.

3. $(x, y, z) \in B \Rightarrow(y, z, x) \notin B,(z, x, y) \notin B$

4. $(x, y, z) \in B$ and $(x, z, t) \in B \Rightarrow(x, y, t) \in B$ and $(y, z, t) \in B$.

Betweenness relations in the setting of $T$-indistinguishability operators have been studied in [9]. Such relations naturally appear if the $t$-norm $T$ is 
Archimedean. In general, indistinguishability operators with respect to nonArchimedean $t$-norms such as $T=M I N$ do not define a betweennes relation on $X$.

Definition 6.2. A T-indistinguishability operator on a set $X$ separates points if $E(x, y)=1$ only when $x=y$.

Proposition 6.3. [9] Let $T$ be an Archimedean continuous t-norm and E a T-indistinguishability operator separating points on a set $X$ and such that $E(x, y) \neq 0$ for all $x, y \in X$. The ternary relation $B_{E}$ on $X$ defined by $(x, y, z) \in B_{E}$ if and only if $x \neq y \neq z \neq x$ and $T(E(x, y), E(y, z))=$ $E(x, z)$ is a betweennes relation.

The condition of separating points (definition 6.2) is closely related to the injectivity of the fuzzy subsets $\left\{h_{i}\right\}_{i \in I}$ involved in the construction of $E$.

Lemma 6.4. Let $h: X \rightarrow[0,1]$ be a fuzzy subset. Then $E_{h}$ separates points on $X$ if, and only if, $h$ is injective.

Proof. Trivial.

Lemma 6.5. Let $T$ be an Archimedean continuous t-norm, and $M$ a quasiarithmetic mean with weights $\left(\alpha_{i}\right)_{i \in I_{-}}$and the same additive generator $t$ as $T$. If $h_{i}$ is injective for all $i \in I$, then $\bar{E}=\underset{i \in I}{M}\left(\alpha_{i} E_{h_{i}}\right)$ separates points.

Proof. Trivial.

Obviously, all of the $h_{i}$ being injective is a sufficient, not necessary, condition for $\bar{E}$ to be separating.

Next proposition establishes how the betweennes relation of $\bar{E}$ is related with those of $E_{h_{i}}$.

Proposition 6.6. Let $T$ be an Archimedean continuous t-norm, $M$ a quasiarithmetic mean with weights $\left(\alpha_{i}\right)_{i \in I}$ and the same additive generator as $T$, $H=\left(h_{i}\right)_{i \in I}$ a family of injective fuzzy subsets of $X$ such that $h_{i}(x)>0$ for all $i \in I$, and $\bar{E}=M_{i \in I}\left(\alpha_{i} E_{h_{i}}\right)$. Then $B=\bigcap_{i \in I} B_{i}$ where $B$ and $B_{i}$ are the betweennes relations of $\bar{E}$ and $E_{h_{i}}$, respectively. 
Proof. First, note that $h_{i}(x)>0$ for all $i \in I$ guarantees both that $E_{h_{i}}(x, y)>$ 0 for all $i \in I$ and that $\bar{E}(x, y)>0$ for all $x, y \in X$, and therefore we may apply proposition 6.3 .

For any given triplet $(x, y, z)$ such that $x \neq y \neq z \neq x$, we have that:

$$
\begin{aligned}
& T(\bar{E}(x, y), \bar{E}(y, z)) \\
& =T\left(M_{i \in I}\left(\alpha_{i}, E_{h_{i}}(x, y)\right), M_{i \in I}\left(\alpha_{i}, E_{h_{i}}(y, z)\right)\right) \\
& =T\left(t^{[-1]}\left(\sum_{i \in I} \alpha_{i}\left(t \circ E_{h_{i}}\right)(x, y)\right)+t^{[-1]}\left(\sum_{i \in I} \alpha_{i}\left(t \circ E_{h_{i}}\right)(y, z)\right)\right) \\
& =t^{[-1]}\left(\left(t \circ t^{[-1]}\right)\left(\sum_{i \in I} \alpha_{i}\left(t \circ E_{h_{i}}\right)(x, y)\right)+\left(t \circ t^{[-1]}\right)\left(\sum_{i \in I} \alpha_{i}\left(t \circ E_{h_{i}}\right)(y, z)\right)\right) \\
& =t^{[-1]}\left(\sum_{i \in I} \alpha_{i}\left(t \circ E_{h_{i}}\right)(x, y)+\sum_{i \in I} \alpha_{i}\left(t \circ E_{h_{i}}\right)(y, z)\right) \\
& =t^{[-1]}\left(\sum_{i \in I} \alpha_{i}\left(\left(t \circ E_{h_{i}}\right)(x, y)+\left(t \circ E_{h_{i}}\right)(y, z)\right)\right) \\
& =t^{[-1]}\left(\sum_{i \in I} \alpha_{i}\left(t \circ t^{[-1]}\right)\left(t \circ E_{h_{i}}(x, y)+t \circ E_{h_{i}}(y, z)\right)\right) \\
& =t^{[-1]}\left(\sum_{i \in I} \alpha_{i} t\left(T\left(E_{h_{i}}(x, y), E_{h_{i}}(y, z)\right)\right)\right)[1]
\end{aligned}
$$

And also that:

$$
\bar{E}(x, z)=t^{[-1]}\left(\sum_{i \in I} \alpha_{i}\left(t \circ E_{h_{i}}\right)(x, z)\right)
$$

In general, [1] $\leq[2]$ because $\bar{E}$ is $T$-transitive.

According to proposition $6.3,(x, y, z) \in B_{i}$, if, and only if,

$$
T\left(E_{h_{i}}(x, y), E_{h_{i}}(y, z)\right)=E_{h_{i}}(x, z)
$$

for all $i \in I$. Also, $(x, y, z) \in B$ if, and only if, $T(\bar{E}(x, y), \bar{E}(y, z))=$ $\bar{E}(x, z)$. 
As a consequence, if $(x, y, z) \in B_{i}$ for all $i \in I$ then [1] $=[2]$,

$$
T(\bar{E}(x, y), \bar{E}(y, z))=\bar{E}(x, z)
$$

and $(x, y, z) \in B$.

Conversely, if $(x, y, z) \in B$ then $T(\bar{E}(x, y), \bar{E}(y, z))=\bar{E}(x, z)$, and therefore $[1]=[2]$. But since $T\left(E_{h_{i}}(x, y), E_{h_{i}}(y, z)\right) \leqslant E_{h_{i}}(x, z)$ for all $i \in I$, the only possibility is $T\left(E_{h_{i}}(x, y), E_{h_{i}}(y, z)\right)=E_{h_{i}}(x, z)$ and then $(x, y, z) \in$ $B_{i}$ for all $i \in I$.

So we have proved that $(x, y, z) \in B_{i}$ for all $i \in I$ if, and only if, $(x, y, z) \in$ $B$, which is equivalent to $B=\bigcap_{i \in I} B_{i}$.

Lemma 6.7. Let $T$ be an Archimedean continuous t-norm, and $h: X \rightarrow$ $[0,1]$ an injective fuzzy subset such that $h(x)>0$ for all $x \in X$. Then for all $x, y, z \in X,(x, y, z) \in B_{h}$ if, and only if, either $x<_{h} y<_{h} z$ or $z<_{h} y<_{h} x$, where $B_{h}$ is the betweenness relation of $E_{h}$, and $<_{h}$ is the order induced by $h$.

Proof. Trivial.

Corollary 6.8. Let $T$ be an Archimedean continuous t-norm, $M$ a quasiarithmetic mean with weights $\left(\alpha_{i}\right)_{i \in I}$ and the same additive generator as $T$, $H=\left\{h_{i}\right\}_{i \in I}$ and $G=\left\{g_{j}\right\}_{j \in J}$ families of injective fuzzy subsets of $X$ such that $h_{i}(x)>0$ and $g_{j}(x)>0$ for all $i \in I, j \in J$ and $x \in X$, and $\bar{E}=$ $M_{i \in I}\left(\alpha_{i}, E_{h_{i}}\right)$ and $\bar{G}=M_{j \in J}\left(\beta_{j}, E_{g_{j}}\right)$ averaged T-indistinguishability operators with betweennes relations $B$ and $D$ respectively.

a. If $\bar{F}=M(\bar{E}, \bar{G})=M_{i \in I}\left(\gamma_{i}, E_{h_{i}^{\prime}}\right)$ for some fuzzy subsets $h_{i}^{\prime} \sim h_{i}$ and weights $\left(\gamma_{i}\right)_{i \in I}$, then $D \subseteq B$.

b. If $\bar{G}=M_{i \in I}\left(\delta_{i}, E_{h_{i}^{\prime}}\right)$, for some fuzzy subsets $h_{i}^{\prime} \sim h_{i}$ and weights $\left(\delta_{i}\right)_{i \in I}$, then $D \subseteq B$.

Proof. Any triplet $(x, y, z)$ such that $(x, y, z) \in D$ but $(x, y, z) \notin B$ satisfies that for all $j \in J$ either $x<_{g_{j}} y<_{g_{j}} z$ or $z<_{g_{j}} y<_{g_{j}} x$, but fails to satisfy both $x<_{h_{i}} y<_{h_{i}} z$ and $z<_{h_{i}} y<_{h_{i}} x$ for at least one $i \in I$. This means that $h_{i} \neq g_{j}$ for all $j \in J$ and therefore neither $M(\bar{E}, \bar{G})=M_{i \in I}\left(\gamma_{i}, E_{h_{i}^{\prime}}\right)$ nor $\bar{G}=\underset{i \in I}{M}\left(\delta_{i}, E_{h_{i}^{\prime}}\right)$ is possible for those $x, y, z$. 


\section{Conclusions and Future Works}

This paper focuses on complex similarity relations that are obtained as quasiarithmetic means of elementary ones induced by single fuzzy attributes. Specifically, we have dealt with the problem of reducing the number of fuzzy attributes used to construct a given complex similarity $\bar{E}$, and solved it by grouping such attributes and averaging them into one single representative per group.

There are some points that need further insight and development. First, we have shown the way to reducing the number of attributes for a particular $\bar{E}$, but no calculation of the minimum possible number of attributes has been provided. This, together with the devising of an explicit algorithm for computing such optimal reduced families of attributes, are important points both for theoretical and practical reasons.

Second, most of the time we have assumed full knowledge of the attributes involved. However, it is not uncommon to be confronted with complex similarity relations as the primitive object to deal with, instead of a set of attributes from which to build on. The attributes have then to be guessed, stated, grouped and aggregated. We have found that betweenness relations provide a starting point to deal with this problem, but further research is needed.

Finally, the devising of a general frame for dealing with all continuous $t$ norms instead of only the Archimedean family and the MIN $t$-norm is needed, for the sake of conceptual simplicity and theoretical completeness. More generally, it would be interesting to reach similar results concerning other structures wich are relevant to applied domains, such as OWA operators, Conjunctors and Overlap Functions [3]. In this respect, the paper [5] provides a starting point by generalising the notion of quasi-arithmetic mean in a direction which looks suitable to our needs. However, the present paper strongly relies on additive generators, and therefore on the metric counterpart of those similarities that are based on Archimedean $t$-norms. All the aforementioned approaches (MIN, OWA and Overlap Functions) lack additive generators, making it difficult to generalise the findings in this paper in a straightforward way. 


\section{References}

[1] J. Aczél. Lectures on functional equations and their applications. Academic Press, NY-London (1966).

[2] D. Boixader, J. Recasens. Characterisation of Unidimensional Averaged Similarities. To appear in Journal of Applied Logics (2017).

[3] H Bustince, J Fernandez, R Mesiar, J Montero, R Orduna. Overlap functions. Nonlinear Analysis: Theory, Methods and Applications 72 (3), 1488-1499, 2010.

[4] P. Hajek. Metamathematics of fuzzy logic. Kluwer Academic Publishers, New York (1998).

[5] J. Jacas, J. Recasens. Aggregation Operators Based on Indistinguishability Operators. Int.J.of Intelligent Systems 21, 857873 (2006)

[6] E.P. Klement, R. Mesiar, E. Pap. Triangular Norms. Kluwer Academic Publishers, Dordrecht (2000).

[7] G. Mattioli, J. Recasens. Structural Analysis of Indistinguishability Operators and Related Concepts. Inf. Sci. 241 (2013) 85-100.

[8] K. Menger. Untersuchungen ber allgemeine Metrik. Math. Ann. 100, 75-113 (1928).

[9] J.Recasens. Indistinguishability Operators. Modelling Fuzzy Equalities and Fuzzy Equivalence Relations. Springer. 2010.

[10] L. Valverde On the Structure of F-Indistinguishability Operators. Fuzzy Sets and Systems 17 313-328, 1985.

[11] L.A. Zadeh. Similarity relations and fuzzy orderings. Inform. Sci. 3, 177$200(1971)$ 